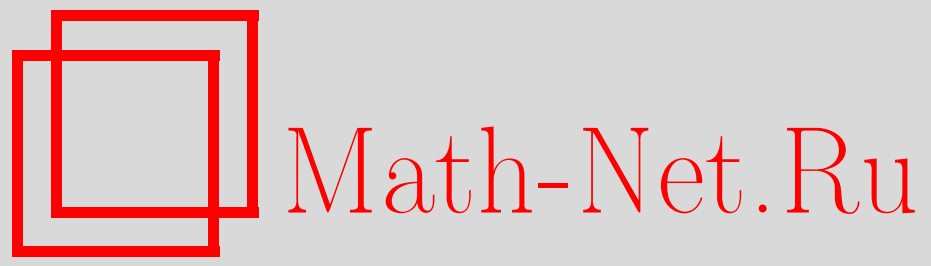

И. М. Гусейнов, Об одном представлении для решения Йоста обыкновенных дифференциальных уравнений, Функи. анализ и его прил., 1999, том 33, выпуск $3,75-77$

DOI: https://doi.org/10.4213/faa370

Использование Общероссийского математического портала MathNet.Ru подразумевает, что вы прочитали и согласны с пользовательским соглашением

http://www . mathnet.ru/rus/agreement

Параметры загрузки:

IP : 3.91 .87 .62

26 апреля 2023 г., 13:28:42

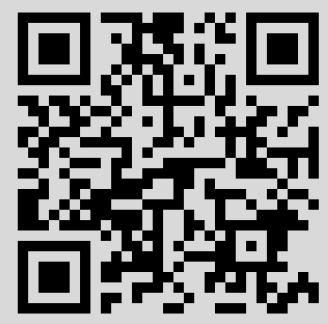


Characteristic Classes, 2-d ed., Gordon \& Breach Science Publ., New York a.o., 1993. Русский перевод: Васильев В. А., Лагранжевы и лежандровы характеристические классы. Изд-во МЦНМО, 1999.

Математический институт им. В. А. Стеклова РАН

Поступило в редакцию 27 апреля 1999 г.

УДК 517.9

\title{
Об одном представлении для решения Йоста обыкновенных дифференциальных уравнений
}

\author{
(C) 1999. И. М. ГУСЕЙНОВ
}

В 1955 г. Б. Я. Левин [1] ввел «треугольное» представление для решения дифференциального уравнения второго порядка, сохраняющего асимптотику в бесконечности, т.е. для решения Йоста. Это представление играло важную роль при решении обратных задач теории рассеяния (см., например, [2]). Для дифференциальных уравнений высокого порядка с аналитическими коэффициентами подобная задача решена в работах [3-5]. Вопрос о необходимости условия аналитичности коэффициентов изучен в [6].

В настоящей работе получены интегральные представления для решения Йоста дифференциальных уравнений высокого порядка, коэффициенты которых могут быть также и неаналитическими функциями. Для описания свойств ядра представления оказалось естественным использование операторов дробного интегрирования и дробного дифференцирования Римана-Лиувилля

$0<\alpha<1$.

$$
I_{t}^{\alpha} \varphi(x, t)=\frac{1}{\Gamma(\alpha)} \int_{0}^{t}(t-s)^{\alpha-1} \varphi(x, s) d s, \quad D_{t}^{\alpha} \varphi(x, t)=\frac{\partial}{\partial t} I_{t}^{1-\alpha} \varphi(x, t),
$$

Несмотря на нетреугольность этих представлений, свойства ядра, в частности, его связь с коэффициентами уравнения, показывают, что их можно использовать в теории обратных задач.

Рассмотрим дифференциальное уравнение $(n>2)$

$$
y^{(n)}+\sum_{j=0}^{n-2} p_{j}(x) y^{(j)}=\lambda^{n} y, \quad x \in \mathbb{R},
$$

где функции $p_{j}(x)$ принадлежат $C^{j}(\mathbb{R})$ и удовлетворяют условиям

$$
\int_{-\infty}^{\infty}(1+|x|)^{n-1-j+s}\left|p_{j}^{(s)}(x)\right| d x<+\infty, \quad 0 \leqslant s \leqslant j \leqslant n-2 .
$$

Введем следующие обозначения:

$$
\begin{gathered}
q_{j}(t)=\sum_{m=j}^{n-2}(-1)^{m} C_{m}^{j} p_{m}^{(m-j)}(t), \\
(J \psi)(x)=\int_{-\infty}^{x} \psi(t) d t, \quad \sigma(x)=\sum_{j=0}^{n-2}\left(J^{n-j}\left|q_{j}\right|\right)(x) .
\end{gathered}
$$


Под $\lambda^{n / 2}$ будем понимать главную ветвь этой функции.

Теорема. Если выполнены условия (2), уравнение (1) для всех $\lambda$ из замкнутого множества $\{\lambda:|\arg \lambda| \leqslant \pi / n\}$ имеет единственное решение $g(x, \lambda)$, удовлетворяющее условию $\lim _{x \rightarrow-\infty} g(x, \lambda) \exp \{-\lambda x\}=1$ и представимое в виде

$$
g(x, \lambda)=e^{\lambda x}\left(1+\int_{0}^{+\infty} G(x, t) e^{-\lambda^{n / 2} t} d t\right)
$$

причем ядро $G(x, \cdot)$ принадлежит $L_{1}(0, \infty)$ и обладает следуюшими свойствами:

(1) $\int_{0}^{+\infty}|G(x, t)| d t \leqslant \exp \{C \sigma(x)\}-1$, где $C$ - некоторая положительная постолннал (см. следствие из леммы 1);

(2) на полуоси $(0,+\infty)$ существуют суммируемые по $t$ производные

$$
\left(D_{t}^{2 / n}\right)^{l} D_{x}^{m} G(x, t), \quad 1 \leqslant l+m \leqslant n-1, l, m=0,1,2, \ldots, n-1,
$$

причем

$$
\lim _{x \rightarrow-\infty} \int_{0}^{+\infty}\left|\left(D_{t}^{2 / n}\right)^{l} D_{x}^{m} G(x, t)\right| d t=0 ;
$$

(3) функиия $G(x, t)$ удовлетворяет уравнению

$$
D_{x}\left(D_{x}^{n-1}+\sum_{l=1}^{n-1} C_{n}^{l}\left(D_{t}^{2 / n}\right)^{l} D_{x}^{n-l-1}\right) G(x, t)+\sum_{l=0}^{n-2} p_{l}(x)\left(D_{t}^{2 / n}+D_{x}\right)^{l} G(x, t)=0
$$

и условиям

$$
\begin{aligned}
& \left.C_{n}^{1} I_{t}^{(n-2) / n} D_{x} G(x, t)\right|_{t=0}+p_{n-2}(x)=0, \\
& \left.\sum_{l=0}^{k-1} C_{n}^{k-l} I_{t}^{(n-2) / n}\left(D_{t}^{2 / n}\right)^{l} D_{x}^{k-l} G(x, t)\right|_{t=0}+p_{n-k-1}(x) \\
& +\left.\sum_{l=0}^{k-2} p_{n-k+l}(x) I_{t}^{(n-2) / n}\left(D_{t}^{2 / n}+D_{x}\right)^{l} G(x, t)\right|_{t=0}=0, \quad k=2, \ldots, n-1 .
\end{aligned}
$$

Приведем схему доказательства.

Рассмотрим интегральное уравнение для решения $g(x, \lambda)$ :

где

$$
g(x, \lambda)=e^{\lambda x}+\sum_{j=0}^{n-2}(-1)^{j+1} \int_{-\infty}^{x} \varphi_{0}^{(j)}(x-t, \lambda) q_{j}(t) g(t, \lambda) d t,
$$

$$
\varphi_{0}(x, \lambda)=\frac{1}{n \lambda^{n-1}} \sum_{m=1}^{n} \alpha^{m} e^{\alpha^{m} \lambda x}, \quad \alpha=e^{2 \pi i / n} .
$$

Подставляя здесь вместо $g(x, \lambda)$ его представление (3) и используя теоремы типа Винера-Пэли для угловых областей (см. [7]), получим следующее уравнение для ядра $G(x, t)$ :

$$
\begin{aligned}
G(x, t)= & \sum_{j=0}^{n-2}(-1)^{j+1} \int_{0}^{+\infty}\left(J^{n-1-j} q_{j}\right)(x-s) \Psi_{j}(s, t) d s \\
& +\sum_{j=0}^{n-2}(-1)^{j+1} \int_{0}^{t} d \eta \int_{0}^{+\infty}\left(J^{n-1-j} q_{j} G(\cdot, \eta)\right)(x-s) \Psi_{j}(s, t-\eta) d s,
\end{aligned}
$$


где

$$
\begin{aligned}
\Psi_{j}(s, t)= & \frac{1}{2 \pi n} \int_{-\infty}^{\infty} e^{i t y} \sum_{m=1}^{n-1}\left(\alpha^{m}\right)^{j+1}\left(\alpha^{m}-1\right)^{n-1-j} \\
& \times \exp \left[\left(\alpha^{m}-1\right)|y|^{2 / n} s \exp (i(\pi / n) \operatorname{sgn} y)\right] d y \quad \text { при } t>0
\end{aligned}
$$

и $\Psi_{j}(s, t)=0$ при $t<0$.

Достаточно доказать, что это уравнение имеет единственное решение $G(x, \cdot) \in L_{1}(0, \infty)$ со свойствами $(1)-(3)$. Последнее устанавливается с помощью следующих лемм.

ЛЕмма 1. При всех $s>0, t>0, j=0,1, \ldots, n-2$ справедливы оченки

$$
\left|\Psi_{j}(s, t)\right| \leqslant C_{0}\left(\frac{s^{n /(n-2)}}{t^{2 /(n-2)}}\right)^{-2 / n}\left[1+\left(\frac{s^{n /(n-2}}{t^{2 /(n-2)}}\right)^{(3 n-4) /(2 n)}\right]^{-1}\left(\frac{s}{t}\right)^{n /(n-2)},
$$

где $C_{0}$ - положительная постоянная, не зависящая от $s, t, j$.

СледСтвиЕ. $0<C \equiv \max _{j} \sup _{s>0} \int_{0}^{\infty}\left|\Psi_{j}(s, t)\right| d t<+\infty$.

ЛЕмма 2. При $t>0$ справедливьи Формульь

$$
\Psi_{j}(s, t)=-\frac{1}{2 \pi n} \sum_{m=1}^{n-1}\left(\alpha^{m}\right)^{j+1}\left(\alpha^{m}-1\right)^{n-2-j} \int_{-\infty}^{\infty} e^{i \xi s} e_{n / 2}\left(t ; \lambda_{m}(\xi)\right) d \xi
$$

$j=0, \ldots, n-2$, дде $e_{\rho}(t ; \lambda)=t^{1 / \rho-1} E_{\rho}\left(-\lambda t^{1 / \rho} ; 1 / \rho\right), a E_{\rho}(z ; \mu)=\sum_{m=0}^{+\infty} \frac{z^{m}}{\Gamma(\mu+m / \rho)}$ - бункиия типа Миттаг-Леффлера [8].

Лемма 3. $D_{t}^{2 / n} e_{n / 2}(t ; \lambda)=-\left.\lambda e_{n / 2}(t ; \lambda) u I_{t}^{(n-2) / n} e_{n / 2}(t ; \lambda)\right|_{t=0}=1$.

Заметим, что при $n=2$ формула (3) совпадает с представлением Б. Я. Левина. При $n>2$ для двучленного уравнения формула $(3)$ с $G(x, \cdot) \in L_{2}(0,+\infty)$ получена в [9]. Там же найдены представления для решения полного уравнения (1), но они отличаются от (3).

В заключение отметим, что аналогичное представление имеет место для решения Йоста уравнения (1), сохраняющего асимптотику лишь в $+\infty$, а также для решения с начальными условиями.

\section{ЛИТЕРАТУРА}

1. Левин Б. Я. ДАН СССР, 106, № 2, 187-190 (1956). 2. Марченко В. А. Операторы Штурма-Лиувилля и их приложения. Наукова думка, Киев, 1977. 3. Хачатрян И. Г. Изв. АН Арм. ССР, сер. матем., 14, №6, 424-445 (1979). 4. Гасылмов М. Г. ДАН СССР, 266, № 5, 1033-1036 (1982). 5. Хачатрян И. Г. Функц. анализ и его прил., 17, вып. 1, 40-52 (1983). 6. Маламуд М. М. Функц. анализ и его прил., 16, вып. 3, 74-75 (1982). 7. Джрбашян M. М., Мартиросян В. М. Изв. АН СССР, сер. матем., 41, № 4, 868-894 (1977). 8. Джрбашян М. М. Интегральные преобразования и представления функций в комплексной области. Наука, М., 1966. 9. Гусейнов И. М. Изв. АН Азерб. CСР, сер. физ.-тех. и матем. наук, № 2, 27-32 (1986).

Бакинский государственный университет

Поступило в редакцию 10 апреля 1997 г. 